\title{
Nota Técnica: \\ Análisis del fenómeno de la expansividad de materiales en túneles construidos en anhidrita. Consecuencias y experiencias
}

\author{
Technical Note: \\ Analysis of the phenomenon of material expansiveness in tunnels built \\ in anhydrite. Outcomes and experiences
}

\author{
J. M. del Campo ${ }^{(*)}$, J. C. Guerra(**), D. Fernández-Ordóñez ${ }^{(*)}$
}

Recepción/Received: 22-IX-11

Aceptación/Accepted: 19-I-12

Publicado online/Online publishing: 4-VI-12

\section{RESUMEN}

Los graves problemas geotécnicos sucedidos en numerosos túneles europeos excavados en materiales que generan problemas de estabilidad, como consecuencia de su expansividad, los convierten en canalizadores de aguas. Estos materiales son fácilmente lavables al disolverse los sulfatos con el agua, provocando una decompresión y remoldeo del macizo, agravando y trasladando el problema más allá del inmediato entorno del túnel. En el caso particular de presencia de capas de anhidrita entre argilitas, además de la adopción de medidas de sostenimiento y revestimiento especiales, el sostenimiento primario y el revestimiento posterior deben ser excepcionalmente resistentes a las presiones que pueden llegar a transmitir el terreno a medio y largo plazo. En este artículo se describen los aspectos más relevantes de comportamiento hidromecánico de los suelos y las rocas sulfatadas y se presentan algunas experiencias, concluyendo sobre aspectos asociados al fenómeno de hinchamiento en túneles y su posible tratamiento.

Palabras clave: yeso, anhidrita, esponjamiento, túneles, modos de fallo.

\section{SUMMARY}

The serious geotechnical problems arising in many tunnels bored in material giving rise to stability problems as a result of being expansive, makes the water channelizers. These are materials which are easily washed with sulphates dissolving with water causing the massif to become decompressed and to remould, aggravating and transferring the problem beyond the tunnel's immediate environment. In the particular case of layers of anhydrite between argillite, it also happens that the primary support and subsequent lining must have exceptional strength to resist the pressures the terrain may transmit in the medium to long term. This article describes the most relevant aspects of the hydromechanical behaviour of sulphated soil and rock and presents some experiments, concluding with various aspects associated to the phenomenon of swelling in tunnels and its possible treatment.

Keywords: gypsum, anhydrite, swelling, tunnels, failure modes.

\footnotetext{
(*) Universidad Politécnica de Madrid (Madrid, España).

(**) Ferrovial - Agroman (Madrid, España).
} 


\section{INTRODUCCIÓN}

La anhidrita es un mineral de composición sulfato cálcico anhidro $\left(\mathrm{CaSO}_{4}\right)$ que al hidratarse se transforma en yeso $\left(\mathrm{CaSO}_{4}+2 \mathrm{H}_{2} \mathrm{O}\right)$. Este proceso físico-químico se esquematiza en la Figura 1, debida a Amstad y Kovari en el año 2001 (1) y a Rauh, Spaun \&Thuro en el 2006 (2). Como se indica, la reacción contempla la disolución y cristalización posterior.

El principal riesgo de la presencia de anhidrita en el túnel viene derivado del potencial hinchamiento de este mineral, en presencia de agua, al transformarse en yeso.

La anhidrita se presenta en rocas sedimentarias, bien en forma de nódulos o agregados cristalinos en el seno de

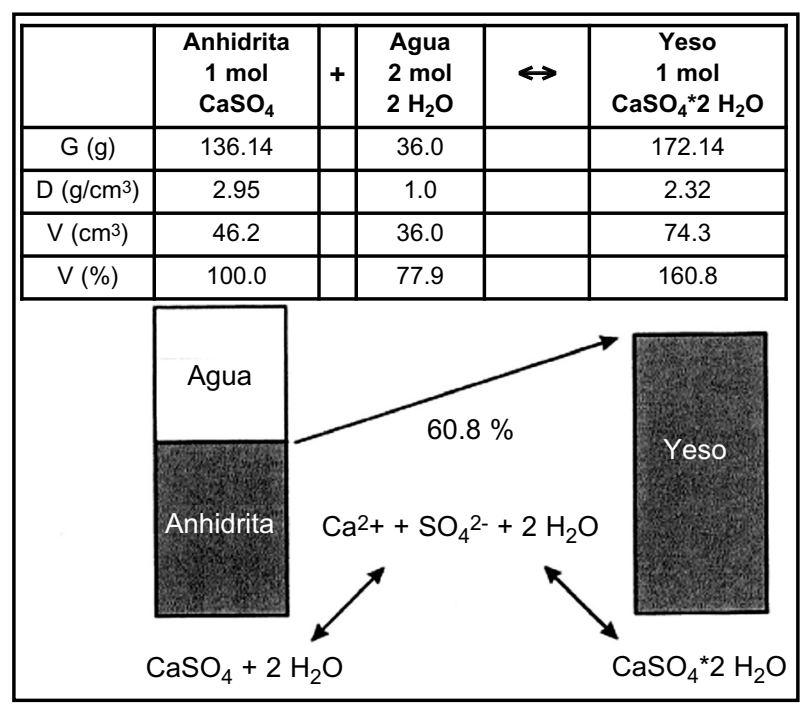

Figura 1. Proceso físico-químico de la transformación de anhidrita a yeso.

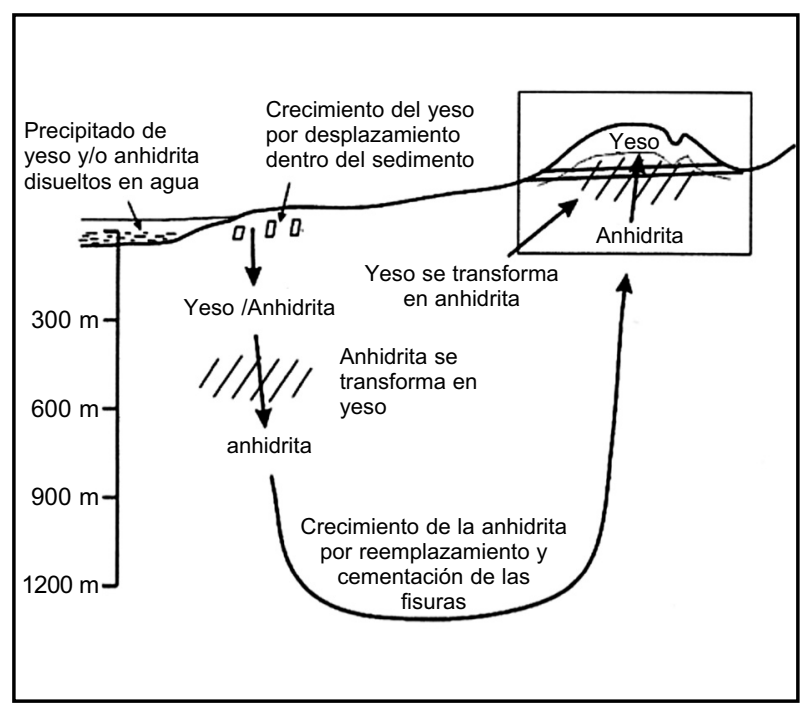

Figura 2. Ciclo "yeso-anhidrita-yeso" según Murray. Ver detalle del recuadro de arriba en la Figura 3. capas de arcillas, yesos o halitas, o bien, en forma de capas masivas interestratificadas normalmente entre materiales arcillosos. También puede aparecer anhidrita en forma de rellenos fisurales o de venas en rocas más competentes, representando en este caso un volumen muy pequeño del macizo rocoso.

El proceso de hidratación de la anhidrita es lento, puede llevar años. No obstante, cuando se trata de interestratificado de arcillas y anhidrita, el proceso puede acelerarse. Ello es debido a que la arcilla facilita la canalización de agua hacia las capas de anhidrita, que son solubles y más permeables, lo que acelera la transformación del sulfato anhidro a sulfato dihidratado, lo que conlleva al hinchamiento de la capa de anhidrita. En cualquier caso, los problemas suelen manifestarse a medio y largo plazo, es decir, cuando el túnel está terminando o incluso en fase de explotación y/o servicio.

De acuerdo a Murray en 1964 (3) y Amstad \& Kovari en el 2001 (1), el esquema de formación de la anhidrita y su presencia a cota de túnel se esquematizan en las Figuras 2 y 3 (2). Podemos observar las áreas de potencial flujo de agua en el túnel.

\section{MATERIALES Y MÉTODOS}

\subsection{El fenómeno de la expansión de la anhidrita}

La interpretación clásica de la expansión de las arcillas expansivas con sulfatos y las rocas sulfatadas se basa en dos mecanismos generales y desacoplados:

a) A corto plazo, si existen arcillas expansivas en el terreno, se produce un hinchamiento físico de las mismas.

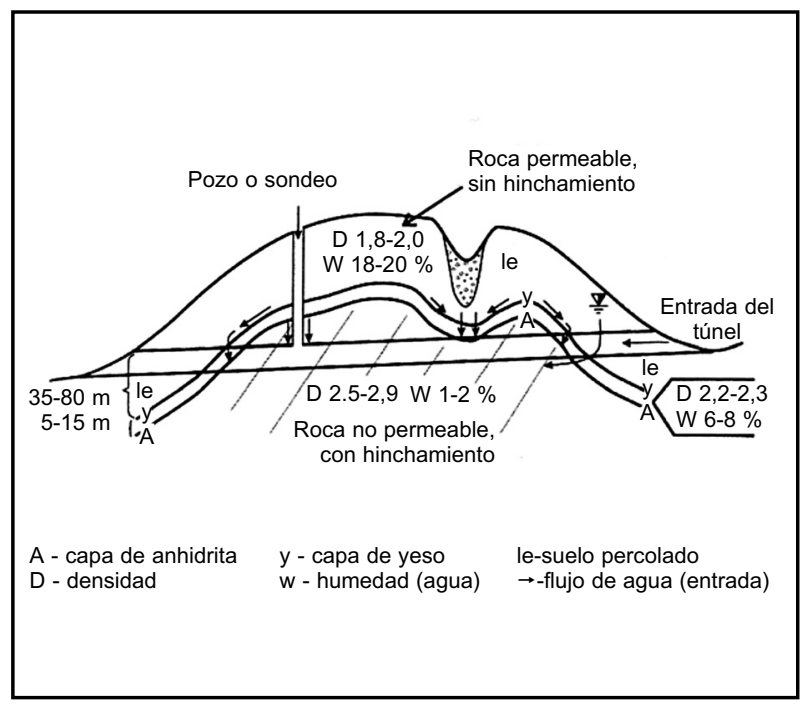

Figura 3. Características de la roca en macizos rocosos que contienen sulfatos. 
b) A largo plazo, se produce un hinchamiento químico debido a la hidratación de la anhidrita para transformarse en yeso, con un incremento volumétrico del $60 \%$. En caso de que el terreno no presentase arcillas (roca de sulfatos cálcicos), este es el único mecanismo actuante.

Algunos autores consideran que el proceso de hidratación de la anhidrita es isovolumétrico (4). Así, la anhidrita se disuelve parcialmente a medida que precipita el yeso secundario, y los excedentes de sulfato cálcico son transportados en solución acuosa, o precipita en fracturas de la roca.

No obstante, otros autores consideran que no pueden darse cambios volumétricos cuando la anhidrita se expone a agua rica en sulfatos, ya que el yeso genera una película que protege la anhidrita (5).

De este modo, la interpretación actual que se da a los procesos de expansión es la siguiente:

- Se continúa aceptando el proceso de expansión de las arcillas a corto plazo.

- A largo plazo, se considera la hidratación isovolumétrica de la anhidrita, y la formación de una fracción acuosa saturada en sulfato cálcico.

- Se asume que la expansión del terreno se produce por precipitación de yeso en fisuras y huecos. Para ello se postulan dos mecanismos:

a) Cuando la temperatura baja por debajo de los $10^{\circ} \mathrm{C}$, se produce la precipitación de los sulfatos en las fisuras del terreno (ver Figura 4).

b) Otro mecanismo de precipitación es la desecación del agua en las fisuras, que provoca la sobresaturación y, por tanto, la precipitación.

En la Figura 4 se muestra la distribución de temperaturas en el Túnel de Lilla (6). En este gráfico se comprueba que solo en invierno pueden alcanzarse marginalmente las condiciones de temperatura necesarias para producir la precipitación. Parece más probable que sea el

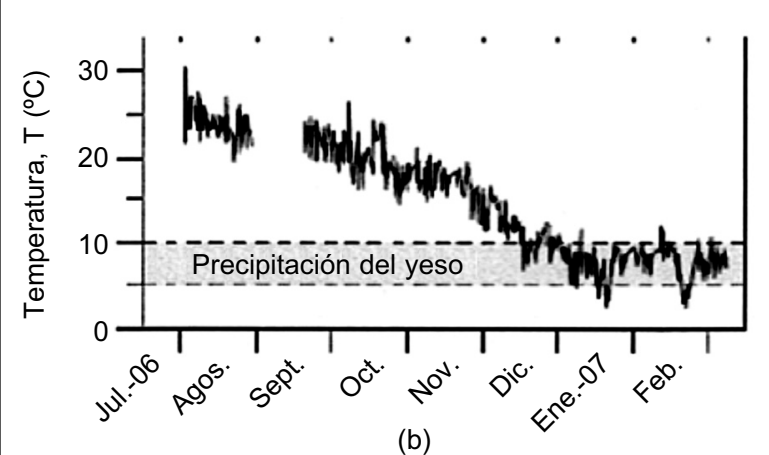

Figura 4. Distribución de temperaturas en el Túnel de Lilla. mecanismo de desecación el causante de la precipitación y, por tanto, de la expansión.

La hidratación de la anhidrita pura conduce teóricamente a una expansión del orden del $60 \%$ en un sistema "abierto" que permita el aporte exterior del agua de reacción. En un sistema "cerrado" (sin aporte de agua exterior), el conjunto anhidrita-agua experimenta una reducción teórica de volumen del $9 \%$ al transformarse en yeso. Las condiciones en las que se desarrolla una obra de excavación son, seguramente, "abiertas" en relación con el flujo de agua.

Ortí y Bayo (4) han estudiado los terrenos evaporíticos del Muschelkalk y Keuper de Tarragona, Ilegando a la conclusión de que ni en el campo, ni al microscopio, se apreciaban signos de hinchamiento de la anhidrita al hidratarse a yeso. Dichos autores citan que este cambio se produce por reemplazamiento sin variación del volumen total de la roca. Es más, literalmente dicen: "En consecuencia, puede pensarse que de cara a problemas relacionados con las obras públicas, la anhidrita podría más bien experimentar reducción de volumen debido a su posibilidad de lenta disolución, que expansiones". También mencionan que la "hidratación de la anhidrita triásica, sin ningún efecto expansivo aparente, está ocurriendo en la actualidad en algunas canteras del Muschelkalk medio del área que nos ocupa".

Tarragó Munté estudió algunos materiales del túnel de LiIla que experimentaron importantes levantamientos de solera (7). Los materiales eran argilitas y margas sulfatadas de la Depresión del Ebro. Este autor menciona que la ejecución de excavaciones en estos materiales suele acompañarse de fenómenos expansivos adicionales a los tradicionales de hidratación de minerales arcillosos. Estos fenómenos causan con frecuencia muchos movimientos en las superficies no confinadas o bien importantes presiones de hinchamiento contra elementos estructurales. Dos aspectos interesantes de estos problemas, dice el autor, son la forma súbita como suelen manifestarse y la manera incontrolada como suelen evolucionar en el tiempo, sin que en algunos casos haya sido posible detectar la estabilización de las expansiones o de las presiones de hinchamiento.

Este mismo autor hace una revisión de las dos teorías enfrentadas sobre la hidratación de la anhidrita. Una, la mencionada anteriormente y defendida por Ortí y Bayo que defiende un proceso de transformación anhidritayeso isovolumétrico en el que la anhidrita se disolvería a medida que precipitaba el yeso secundario. La segunda teoría de la transformación yeso-anhidrita la considera como una reacción química reversible condicionada por la temperatura, la concentración de sales del agua, la presión de confinamiento y la naturaleza "abierta" o "cerrada" del medio donde tenga lugar el proceso. 
De modo ilustrativo en la Figura 5 se muestran las imágenes tomadas mediante microscopio de barrido electrónico de una misma muestra de anhidrita masiva inalterada en principio (izquierda) y de la anhidrita alterada (derecha) después, en las que puede apreciarse el incremento de volumen sufrido de cada partícula mineral. Ambas imágenes corresponden a materiales extraídos del túnel de Lilla, en Montblanc, Tarragona.

\subsection{La cuantificación del problema: ensayos de campo y de laboratorio}

Para realizar el análisis detallado del problema que plantea la existencia de anhidritas en el macizo rocoso que tiene que atravesar un túnel resulta obligada la realización de una serie de ensayos de campo y de laboratorio encaminados a determinar la presión de hinchamiento máxima que pudieran presentar estos materiales, con el fin de dimensionar una sección de túnel que responda a las solicitaciones del material excavado.

A este respecto, citar que no resulta fácil la determinación del hinchamiento libre y de las presiones que se van a generar en el transcurso del fenómeno expansivo. Se requieren largos plazos de ensayos en campo o sobre muestras y, desgraciadamente, normalmente no se dispone del tiempo suficiente a la hora de elaborar los proyectos.

Los ensayos más fiables en campo son la realización de secciones instrumentadas sobre galerías de reconocimiento. El problema de esta técnica es que requiere mucha antelación y paciencia, además del importante esfuerzo económico que conlleva. Donde más se ha usado y se conocen muchos de los resultados es en Alemania.

En laboratorio, las pruebas se basan en el edómetro pero, igualmente, requieren la condenación de costosos equipos durante años para tener datos, especialmente si se trata de anhidrita pura. La consulta de la información existente sobre el tema concluye en la conveniencia de realizar dos tipos de ensayos: ensayo de presión de hinchamiento y el ensayo de inundación bajo carga constante (método Huder-Amberg). Estos ensayos permiten cuantificar los posibles cambios de volumen que pueden experimentar los suelos frente a los cambios de humedad (8). A continuación se realiza una breve descripción de cada uno de estos ensayos.

\subsubsection{Ensayo de presión de hinchamiento}

Este ensayo se realiza para medir la expansividad de un suelo parcialmente saturado. El ensayo consiste, básicamente, en impedir el aumento de volumen de una muestra inalterada o remoldeada de suelo, colocada en una célula edométrica e inundada, mediante la aplicación de incrementos de carga verticales .Cuando no se aprecia tendencia en la variación del volumen de la probeta con el tiempo, se considera que se ha alcanzado el equilibrio y se da por finalizado el ensayo. Se denomina, por tanto, presión de hinchamiento a la presión vertical necesaria para mantener sin cambio de volumen una probeta confinada lateralmente cuando se inunda de agua.

Posteriormente se procede a descargar por escalones. Los resultados obtenidos se expresan en un gráfico en el que se representan los tantos por ciento de hinchamiento y la presión que actúa sobre la muestra en cada escalón de descarga.

Para la determinación de la presión de hinchamiento se debe atender la norma UNE 103602 (ensayo para calcular la presión de hinchamiento de un suelo en edómetro). Las muestras son probetas cilíndricas de $20 \mathrm{~mm}$ de altura y $50 \mathrm{~mm}$ de diámetro, aproximadamente. En muestras de suelos se aplica una presión inicial en el edómetro, no

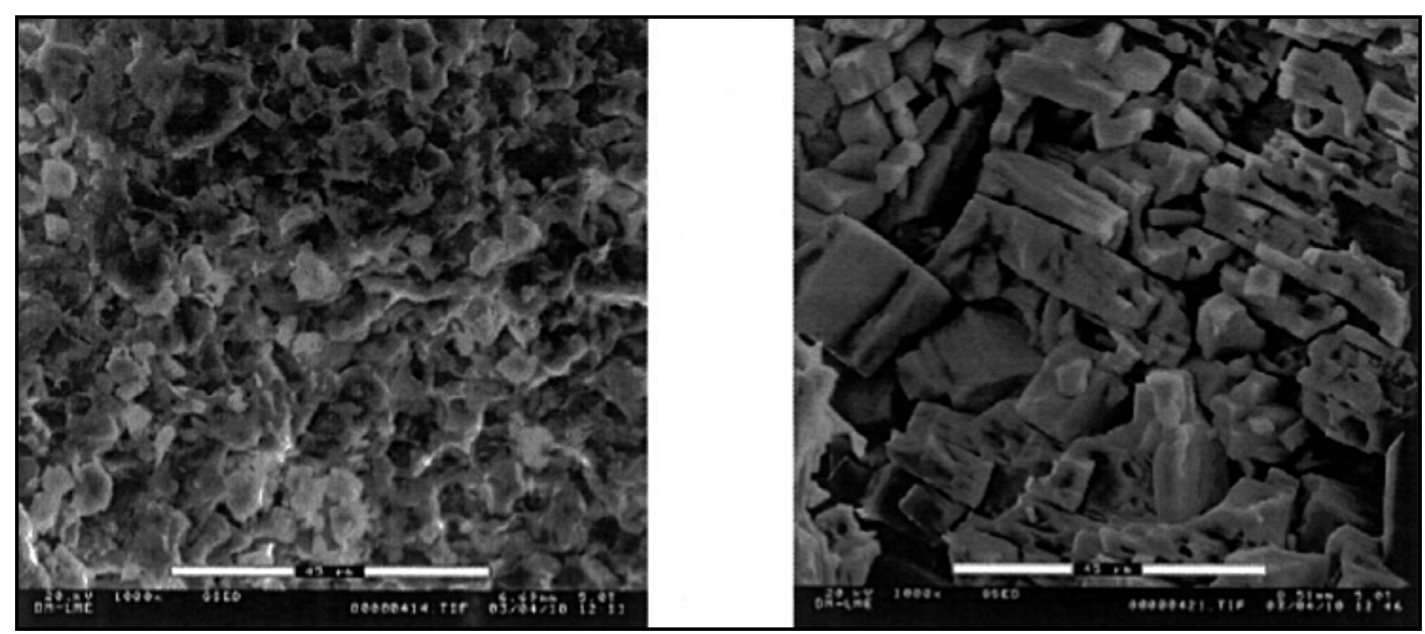

Figura 5. Imágenes de una muestra de anhidrita inalterada (izquierda) en principio y alterada (derecha) más tarde a yeso. 
así en rocas. Normalmente, sucede que como la duración de los ensayos es corta (las habituales prisas inherentes a los proyectos y a las obras) no se puede asegurar que se alcance el equilibrio. Por lo tanto, no se suele efectuar el proceso de descarga y, por lo tanto, no se puede representar gráficamente la relación entre los tantos por ciento de hinchamiento y la presión que actúa sobre la probeta en cada escalón de descarga, aspecto que suele resultar muy deseable y conveniente para una correcta ejecución del ensayo.

A este respecto, citar que algunas referencias bibliográficas consultadas sobre ensayos de hinchamiento en rocas ricas en minerales sulfatados y con minerales arcillosos mencionan ensayos de una duración superior a los 1.000 días (9). Es posible que si el ensayo fuese más largo la presión de hinchamiento resultase más elevada. En algunas de las referencias bibliográficas consultadas se habla de aparición de presiones de hinchamiento después de 600 días.

\subsubsection{Inundación bajo carga constante ("standard Huder-Ambergswelling test")}

Este método combina el ensayo de presión y el de deformación de hinchamiento del suelo, que se observa en la Figura 6.

Después de la primera carga $(A)$, una descarga (B) y una recarga $(C)$, se sube al siguiente escalón de presión hasta que el asentamiento o el desplazamiento varíen. Después se añade agua destilada para iniciar el proceso de hinchamiento (E). El asentamiento (D) ocurre porque la muestra en polvo consolida en contacto con el agua bajo una elevada presión axial.

El hinchamiento libre se determina según las recomendaciones de la ISRM (1981). Los ensayos se efectúan sobre probetas cilíndricas de alturas comprendidas entre 60 y $70 \mathrm{~mm}$ y con un diámetro de $50 \mathrm{~mm}$. La precisión de las medidas de hinchamiento suele ser de $1 \mu \mathrm{m}$. El

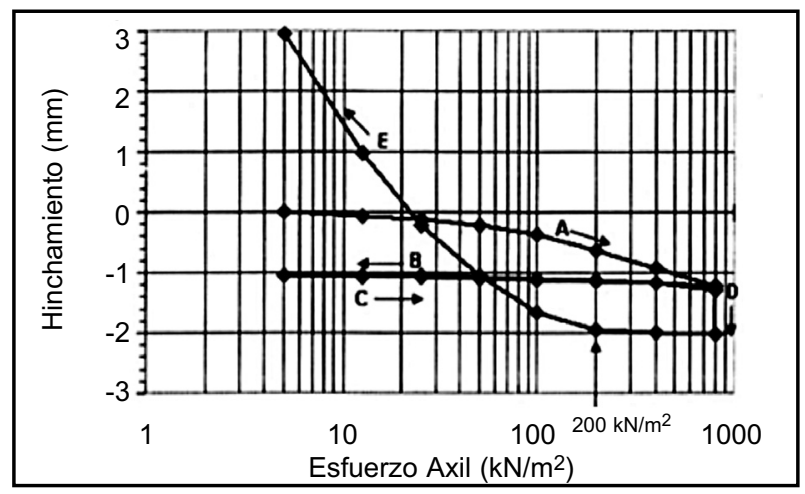

Figura 6. Gráfica típica del ensayo de inundación bajo carga constante. cálculo del coeficiente de hinchamiento se realiza mediante la fórmula [1]:

$$
\varepsilon_{\mathrm{S}}=100 \mathrm{x} \Delta \mathrm{l} / \mathrm{I}(\%)
$$

donde, "I" es la longitud inicial de la probeta seca y " $\Delta \mathrm{l}$ " la expansión máxima alcanzada.

El hinchamiento puede determinarse también según las recomendaciones de la ISRM. Mediante este ensayo se registran simultáneamente en tres direcciones perpendiculares las deformaciones longitudinales producidas en las probetas, libres de restricciones laterales, cuando están sumergidas en agua, como se muestra en la Figura 7. El coeficiente de hinchamiento se calcula mediante la misma fórmula [1]. Los ensayos se efectúan sobre probetas cilíndricas de $50 \mathrm{~mm}$ de diámetro y alturas comprendidas entre 70 y $100 \mathrm{~mm}$. La precisión de las medidas de desplazamiento es, en general, inferior a $1 \mu \mathrm{m}$.

\section{RESULTADOS Y DISCUSIÓN}

\subsection{Las consecuencias de la expansión}

Las deformaciones en un túnel por efecto del hinchamiento se producen frecuentemente con el levantamiento de la solera y las convergencias (abombamientos) de los hastiales como se observa en la Figura 7 (10). Debido a la heterogeneidad intrínseca de las rocas sulfatadas, es frecuente que exista una gran anisotropía en el macizo y que, por tanto, los desplazamientos y las presiones tengan lugar de manera muy heterogénea. La rotura de los elementos estructurales aparece, especialmente, en la solera, aunque la casuística de los efectos del hinchamiento sobre el revestimiento del túnel es amplia y compleja.

En la bibliografía pueden consultarse numerosos ejemplos y estudios realizados por diversos autores acerca de los daños y roturas en túneles excavados en rocas triásicas tanto del Keuper Medio (Gipskeuper) como del

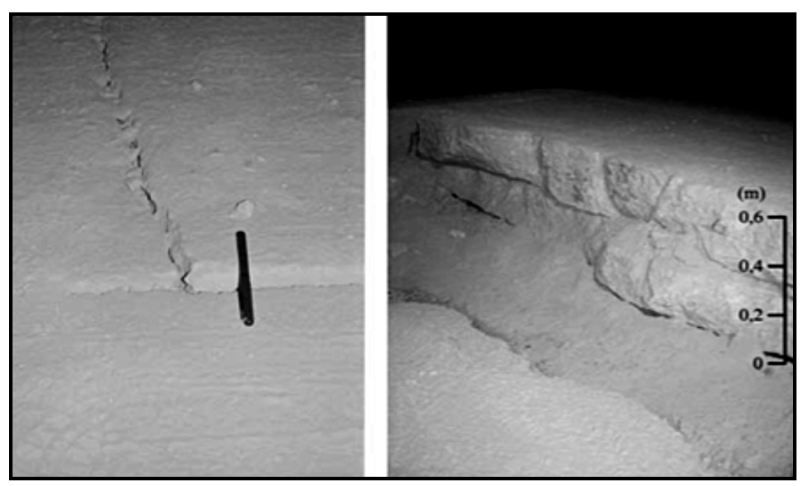

Figura 7. Túnel de Lilla. Véase el levantamiento de solera en PK 411+880 en marzo de 2003 (izquierda) y en septiembre de 2003 (derecha). 
Muschelkalk Medio (Anhydritgruppe), en Baden-Württemberg (Alemania) y Jura Mountains (Suiza). Algunos de los trabajos más reseñables son los realizados por Huder y Amberg en 1970 (8), Henke et al. en 1975 (11), Steiner en 1993 (9), Paul y Wichter en 1996, Amstad\&Kovári en 2001 (1) y Wittke en 2006. Además, se han observado varios casos de fenómenos expansivos en excavaciones profundas y túneles en rocas blandas arcillosas sulfatadas de la cuenca terciaria del río Ebro, estudiados, entre otros, por Serrano et al. en 1981 (12) y 1988 (13), Lloret et al. en 1988 (14), Alonso et al. en 2004 (15, 16), en 2005 (17) y en 2007 (18), Deu \& Tarragó en 2004 (19), Tarragó et al. en 2005 (20), Alonso \& Berdugo en 2006 (21) y 2007 (6) y Berdugo et al. en el año 2007 (10), estos últimos con especial atención a los fenómenos expansivos observados durante la construcción de tres túneles de la nueva Línea de Alta Velocidad Madrid-Barcelona-Frontera Francesa. Todos estos trabajos han contribuido al conocimiento de los procesos expansivos que suelen ocurrir en estructuras excavadas en contextos arcillosos sulfatados. En los gráficos de la Figura 8 se resumen algunas de estas experiencias (22).

Las presiones de hinchamiento mencionadas en dichos artículos relacionados con túneles en materiales arcillosos y sulfatados han dado valores que oscilan entre 0,0 y 5,5 MPa. A modo de ejemplo, en la Tabla 1 se exponen los rangos de valores de la presión de hinchamiento en los casos más significativos (23):

En el gráfico de la Figura 8 y en laTabla 1 puede comprobarse que los principales problemas que se han producido en túneles excavados en el sur de Alemania y norte de Suiza es que han atravesado rocas sulfatadas de las facies Keuper y Muschelkalk del periodo Triásico. Concretamente, el Túnel de Wagenburg es el que históricamente ha sufrido las mayores presiones, alcanzando valores del orden de 5,5 MPa. En España, el caso más importante se ha dado en el mencionado Túnel de Lilla, con presiones de hinchamiento del orden de $5 \mathrm{MPa}$.

Por otra parte, la distinción entre "rocas sulfatadas" y "otros materiales expansivos" (arcillas y margas) pone de manifiesto que, bajo condiciones de construcción y servicio similares, el hinchamiento libre y las presiones de hinchamiento en rocas sulfatadas pueden ser un orden de magnitud mayores que en los materiales arcillosos. Además, a partir de los resultados y datos observacionales obtenidos en algunas galerías de prueba en Alemania y Suiza, parece ser que el hinchamiento en túneles excavados en rocas sulfatadas crece continuamente

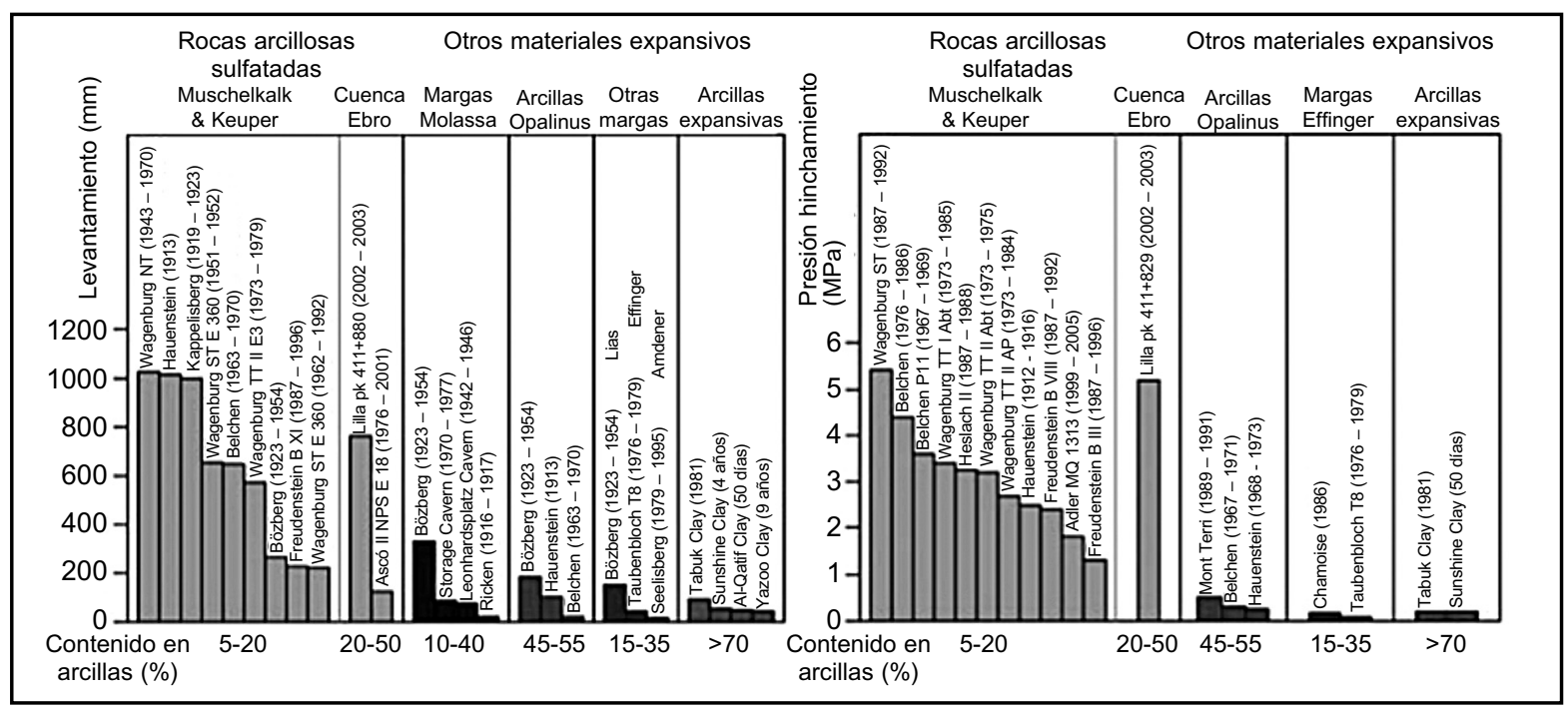

Figura 8. Algunos ejemplos de levantamientos de túneles con fenómenos expansivos. Observaciones de campo de fenómenos expansivos extremos en túneles, cavernas, excavaciones profundas y cimentaciones en rocas expansivas y suelos.

Tabla 1

Presiones máximas de hinchamiento (Mpa) de algunos túneles ejecutados en rocas arcillosas sulfatadas.

\begin{tabular}{|c|c|}
\hline Túnel & Presión máxima de hinchamiento \\
\hline Wagenburg ST (Alemania) & 5.5 \\
\hline Lilla (España) & 5.0 \\
\hline Belchen (Alemania) & 4.5 \\
\hline Heslach II (Alemania) & 3.5 \\
\hline Hauenstein (Alemania) & 2.5 \\
\hline
\end{tabular}


y, en algunos casos, no se puede definir el valor máximo de dichas presiones de hinchamiento.

En el túnel de Lilla los fenómenos expansivos se produjeron a nivel de solera (hasta de $80 \mathrm{~cm}$ de levantamiento), pero no se han atribuido a la transformación de anhidrita en yeso, sino a la expansión de la arcilla y a la cristalización de minerales sulfatados en discontinuidades, que ejercerían un efecto de cuña. (10), en su trabajo concluyen que la precipitación de los yesos y de otros tipos de sulfatos hidratados resulta termodinámicamente posible bajo unas adecuadas condiciones de humedad relativa en el interior del túnel, por lo que es posible el crecimiento de los cristales de sulfatos en las discontinuidades del macizo rocoso. Además, otros autores como Tarragó Munte (7) mencionan que, en los ensayos de hinchamiento realizados en el laboratorio, las muestras con arcillas sí hinchaban, pero las que estaban formadas por anhidrita no lo hacían.

De acuerdo a Tarragó, el mecanismo de expansión actuante en el Túnel de Lilla fue el expuesto en la Figura 9.

En resumen, Tarragó asume en el Túnel de Lilla un papel muy importante a las fisuras provocadas por las voladuras,

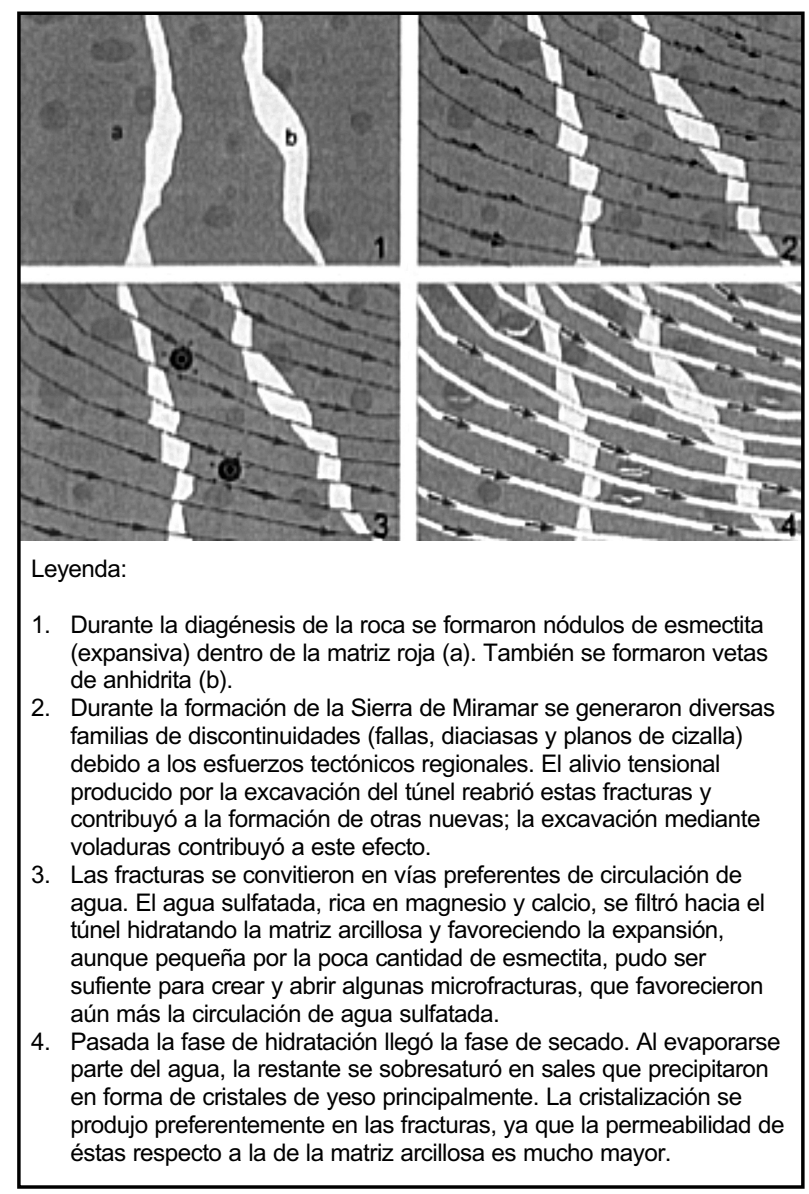

Figura 9. Modelo conceptual de los hinchamientos en las argilitas sulfatadas del macizo de Lilla. al efecto acoplado de formación de microfisuras por expansión de las esmectitas, a las diaclasas pre-existentes y a las nuevas producidas por alivio tensional tras la excavación. Los problemas de expansión en este caso parecen haber sido debidos más al hinchamiento de las arcillas expansivas que al de la transformación de anhidrita en yeso.

Otros casos conocidos de este problema acaecidos en Europa son los siguientes:

- El Túnel de UPPER HAUENSTEIN, túnel ferroviario que se construyó hace más de 150 años, su perfil transversal se redujo considerablemente debido a levantamientos de solera y abombamientos laterales, que condujeron a la reducción del número de vías de dos a una. Las deformaciones se originaron en margas del Dogger inferior, destruyéndose la contrabóveda. Durante cuatro años se midieron levantamientos de $11 \mathrm{~mm} / \mathrm{año}$.

- El Túnel de HAUSENTEIN BASE (de 8.134 m de longitud) tenía en el año 1916, 624 m de túnel con contrabóveda. Una primera reconstrucción en los años 1919-1923, demostró que la contrabóveda era necesaria en 2.294 m. Una segunda reconstrucción durante los años 1980-1986 incrementó nuevamente la longitud el tramo con contrabóveda a un total de $5.386 \mathrm{~m}$ (66\% de la longitud del túnel). Todas las zonas con argilitas tenían contrabóveda. Medidas tomadas en análisis retrospectivos dieron valores de presión de hinchamiento de 0,2 a 0,3 MPa en las arcillas opalinas.

- El Túnel de CZERNITZER es uno de los ejemplos más impresionantes de levantamiento de solera. Se construyó en alternancias finas de sulfatos y argilitas. El túnel se deformó completamente en dos semanas. El incremento de volumen y el método de excavación fueron los responsables conjuntamente. El levantamiento de la contrabóveda llevó asociada la convergencia de los hastiales. El inicio del proceso se manifestó en las acciones siguientes: pérdida completa de la contrabóveda, fallo de apoyo o levantamiento, transformación de la anhidrita a yeso con aumento de volumen (60\%) y arcillas expansivas (adsorción de agua).

- El Túnel de BöZBERG (de 2,5 km de longitud) se construyó entre 1871 y 1875 , sufriendo considerables levantamientos y movimientos laterales debido a la presencia de arcillas expansivas opalinas y también de anhidritas. Se midieron importantes levantamientos de solera (hasta $45 \mathrm{~cm}$ ), convergencias de hastiales $(13 \mathrm{~cm})$ y ascensos de clave $(5 \mathrm{~cm})$. Varios tramos debieron ser reconstruidos entre 1903 y 1905 realizándose una contrabóveda que sería destruida por aguas sulfatadas. Además, como consecuencia de tales deformaciones, los drenajes se rompían y ayudaban a realimentar el proceso. 
- El Túnel de KAPPELESBERG se construyó entre 1878 y 1980, en margas, yesos y anhidritas. Debía alojar dos vías pero, debido a las fuertes deformaciones, solo pudo instalarse una vía. La solera se levantaba durante la construcción unos $25 \mathrm{~cm} /$ año y se rebajó hasta 26 veces. Esta medida fue muy perjudicial, ya que permitió la progresiva hidratación de las anhidritas. En años posteriores los movimientos de solera oscilaron entre 23 y $52 \mathrm{~mm} /$ año. El valor total del levantamiento llegó a los 4,7 m. Entre 1982 y 1983 se reconstruyó el túnel casi por entero excavándose una contrabóveda (sección final circular) y colocándose otro sostenimiento sobre el existente.

- El Túnel de WAGENBURG, que se construyó entre 1942 y 1957, tiene una longitud de $824 \mathrm{~m}$. Se excavó en arcillas de keuper con yesos y anhidritas. El nivel de yeso se encontraba a una profundidad de 30 a $50 \mathrm{~m}$ bajo la superficie. El levantamiento de la solera estaba restringido a los $200 \mathrm{~m}$ más internos donde, posiblemente, se cortaba un nivel de anhidritas o su contacto estaba muy próximo a la solera del túnel. Se observó el levantamiento de todo el túnel en la zona de transición yeso-anhidrita, lo cual se tradujo en un levantamiento de la superficie topográfica. Las medidas se tomaron tanto en el túnel principal (construido por canal de ventilación, tubo de circulación y canal de drenaje), como la galería de experimentación $(2,7 \mathrm{~m} \mathrm{x} \mathrm{3,0} \mathrm{m).} \mathrm{Entre} 1942$ y 1970 se ha medido un levantamiento máximo de $102,9 \mathrm{~cm}$ con velocidades de 50 a $60 \mathrm{~mm} / a n ̃ o$. En el canal de ventilación se midieron $9,3 \mathrm{~cm}$ en 12 años. Por otro lado, todo el túnel se levanta a una velocidad de 0,7

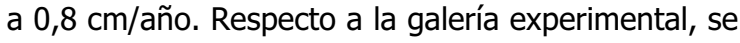
ha medido un levantamiento de la clave de $30 \mathrm{~cm}$ en 32 años. A partir de 1982 la velocidad del levantamiento de la contrabóveda ha sido de $7 \mathrm{~mm} / \mathrm{año}$ y, finalmente, tuvo que ser reconstruida en 1987.

- El Túnel de BELCHEN (de 3,2 km) fue construido entre 1963 y 1970, presentando fuertes levantamientos en los tramos de formaciones de Keuper y arcillas opalinas. El método de construcción adoptado, excavando dos galerías laterales, permitió la entrada de agua a la solera. El valor del levantamiento se incrementó considerablemente a $90 \mathrm{~cm}$, tras la excavación, en pocos meses. Las zonas dañadas se remplazaron por una contrabóveda diseñada para presiones de 0,85 MPa. Sin embargo, la ocurrencia de mayores presiones (de 1,0 a 1,2 Mpa) dañaron la contrabóveda en los lados al concentrarse los esfuerzos en las esquinas del sostenimiento. Las deformaciones fueron de $60 \mathrm{~cm}$, originadas en margas negras con venas muy finas de anhidrita. En este caso las margas actuaron como conductoras de la humedad, poniéndola en contacto con las venas de anhidrita que se transformaron a yeso. En las células de presión se han obtenido presiones de hinchamiento máximas de 0,3 $\mathrm{MPa}$, con una media de 0,17 MPa. En ensayos de laboratorio se han obtenido valores de 0,8 a 2,0 MPa. Se reconstruyó la contrabóveda con una curvatura apropiada $(R=8,12 \mathrm{~m})$, se incrementó su espesor en $85 \mathrm{~cm}$ y se reforzó con armadura. Se instalaron células de presión entre el sostenimiento y el terreno, y dentro del hormigón. Los resultados fueron los siguientes: presión máxima de la roca: 3 Mpa; tensión máxima en hormigón: $27 \mathrm{MPa}$. La deformación ha sido medida mediante la instalación de bulones en todas las secciones. Su desarrollo también es lineal. Las deformaciones pueden transformarse a tensiones multiplicando por el módulo de elasticidad. Así, se obtuvieron valores entre 15 y $20 \mathrm{MPa}$.

- En el Túnel T8, en Suiza, los valores de laboratorio variaron entre 0,0 y 0,8 MPa (media de 0,4 MPa). Durante la construcción cesó el levantamiento de la solera bajo una sobrecarga de $60 \mathrm{KPa}$. Para el sostenimiento final se colocó un nivel compresible debajo de la contrabóveda, permitiendo que la base cediera. Se calculó por extrapolación un levantamiento de 20 a $110 \mathrm{~mm}$.

- En el Túnel de MONT-TERRI, en la galería piloto, se midieron 0,3 MPa en un año en las argilitas del keuper y 0,5 MPa en las arcillas opalinas, en dos años. En el Oxfordiense, se midieron 0,7 MPa (2 años). Solo se observó hinchamiento en la contrabóveda.

- Para construir el Túnel de CHAMOISE (Francia), se realizó un programa de testificación y monitorización. Los ensayos de laboratorio dieron presiones de hinchamiento entre 3 y $8 \mathrm{MPa}$. En una sección del túnel piloto, se estudió el efecto de colocar el sostenimiento cerca del frente. En el caso de un sostenimiento con gunita, se midió una tensión radial de 2,5 MPa. Cuando el sostenimiento se colocaba después de producirse una redistribución de tensiones, se midieron solo 0,6 MPa de tensión radial (medida de convergencias). En el túnel principal, se permitió una redistribución de tensiones. Las convergencias diametrales dieron valores comprendidos entre 5 y $45 \mathrm{~mm}$, indicando un cierre asimétrico del túnel. Las tensiones de hinchamiento calculadas en análisis retrospectivos, después de algunos años, arrojaron valores de tan solo 0,15 MPa.

- El Túnel de SELISBERG atraviesa rocas del cinturón alpino (arcillas Amdener y arcillas de Balanging). Ambas unidades rocosas tienen propiedades geomecánicas similares, pero se emplearon diferentes métodos de excavación y secciones distintas. En la sección Norte (arcillas Amdener) se utilizó un sistema de excavación convencional con perforación y voladura. En la contrabóveda se observaron fenómenos de hinchamiento tardíos. En la sección central (arcillas de Valangin), se utilizó una sección circular que se colocó de forma inmediata mediante dovelas prefabricadas detrás de un escudo que realizaba la excavación. 
No se han dado hinchamientos. Por lo tanto, se deduce también que la forma geométrica de la sección del túnel y el método de excavación empleado juega un papel importante en el fenómeno del hinchamiento.

- En el túnel ferroviario de SAN DONATO (Italia), la galería Sur, descendente, experimentó hinchamientos muy fuertes con convergencias radiales de $1 \mathrm{~m}$. La galería Norte, ascendente, tuvo convergencias del orden de decímetros. Cuando se comparó la composición mineralógica de las rocas, se observó que las arcillas de la galería Norte contenían más minerales expansivos, de modo que tenía, teóricamente, un potencial expansivo más alto que aquellas rocas de la galería Sur. Pero no fue así. La explicación se debe a que en la galería Sur, descendente, el agua procedente de una formación de areniscas podía influir y concentrarse en el fondo de la galería. La arcilla recién excavada estaba así sometida a una meteorización continua por hidratación, además de las presiones de succión generadas en la arcilla sin carga litostática, que propiciaba una alimentación continua. El fenómeno de hinchamiento estaba relacionado con el acceso del agua y la alimentación continua. Por tanto, no solo es necesario tener un material expansivo para producir hinchamientos, sino también agua que acceda de forma continua para producir la hidratación necesaria.

\subsection{Tratamiento de zonas de anhidrita. Técnicas constructivas}

Los criterios más generales para diseñar secciones capaces de resistir el empuje de la anhidrita son:

- Secciones lo más circulares posibles.

- Colocación de contrabóveda robusta.

- Permitir cierto hinchamiento del terreno con objeto de reducir los empujes sobre el sostenimiento.

- Aplicar el sostenimiento-revestimiento lo más cerca posible del frente.

- Limitar la entrada de agua al terreno.

- Evitar la degradación o plastificación del terreno que acompaña al hinchamiento, sobre todo en caso de fuertes tensiones geostáticas.

En la Figura 10 se presentan algunas secciones empleadas en diversos túneles de Alemania. De acuerdo con Wittke (24), la principal ventaja del diseño del sostenimiento-revestimiento rígido para túneles en rocas sulfatadas es el autosellado inducido por el propio hinchamiento alrededor del túnel.

En cuanto al criterio de permitir ciertos hinchamientos se producen dos tendencias claramente diferenciadas:

- Permitir el desarrollo de hinchamientos: se consigue dejando zonas huecas debajo de la solera, colocando

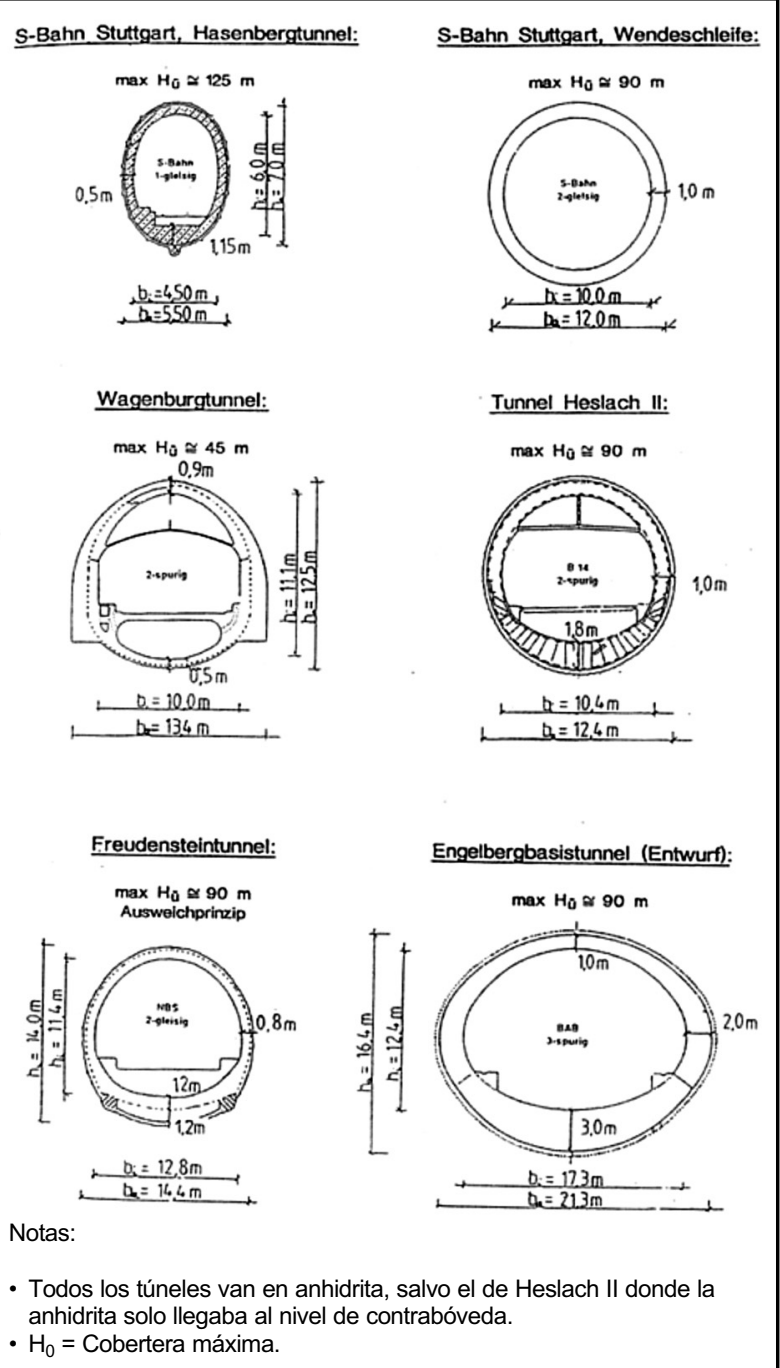

Figura 10. Secciones típicas utilizadas en Alemania para resistir el empuje de la anhidrita.

materiales con huecos como gravas, y mediante materiales de poca densidad con fácil capacidad para comprimirse. Esto se ha empleado en el pasado, en los primeros diseños, estando actualmente en desuso por presentar problemas de deformaciones asimétricas, y no impedir la entrada de agua al macizo, incluso favoreciéndola, produciendo un efecto negativo que se prolonga en el tiempo.

- Impedir cualquier hinchamiento resistiendo las presiones que se produzcan: requiere un refuerzo de la bóveda y especialmente el empleo de una contrabóveda muy gruesa, buscándose una sección circular. Este tipo de soluciones se han visto potenciadas en su uso, además de por mejores resultados, por el avance en los métodos de cálculo.

En general, las técnicas constructivas con las que se han abordado tradicionalmente dichos túneles suponían actuar sobre la zona de contrabóveda de la sección. En la 


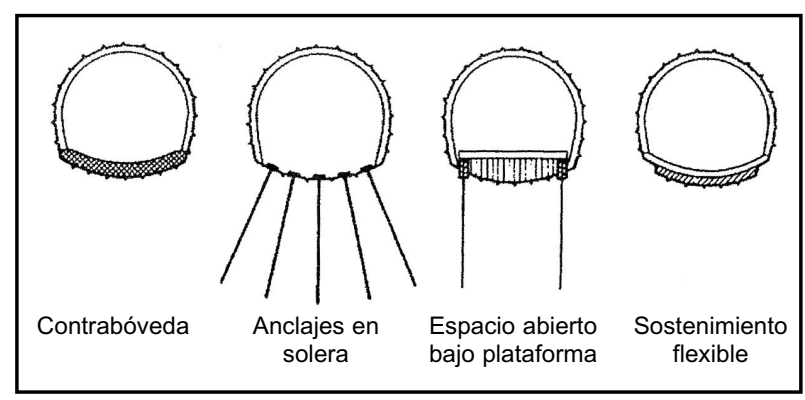

Figura 11. Soluciones constructivas en contrabóveda de túnel.

Figura 11 se ilustran algunos ejemplos de tratamientos constructivos adoptados en túneles con expansividad de anhidritas (25). Como puede observarse, los principales tratamientos que se aplican son:

- Aplicación de contrabóveda gruesa, de hormigón armado.

- Aplicación de anclajes en solera.

- Dejar un espacio abierto bajo la plataforma, que sirve de cámara de expansión libre, evitando que la expansión presione el revestimiento.

- Sostenimiento flexible.

La experiencia alemana muestra que, en general, estos túneles se excavan con facilidad, aunque sea necesario incluso el empleo de voladuras, y mostrando una buena estabilidad a corto plazo. Los problemas se desarrollan en el medio y largo plazo, a medida que se desarrolla el fenómeno de la expansión.

Pronto se comprobó que las secciones tipo que dejaban un espacio para la expansión del terreno no funcionaban adecuadamente, ya que era cuestión de tiempo que la plataforma sufriera la presión del terreno. En las soluciones con anclajes, se comprobó que a la larga estos perdían carga, y el revestimiento comenzaba a sufrir la presión de la expansión del terreno. En el caso de los sostenimientos flexibles, con el tiempo llegaban a deformarse tanto que permitían la entrada de filtraciones y era necesaria su reparación.

De las soluciones aplicadas, las que mejores resultados han presentado es la aplicación de revestimientos muy rígidos, de hormigón armado, dotados de contrabóveda muy robusta. Estos revestimientos adquirían unas cargas notables, pero estaban diseñados para soportarlas. Se comprobó que a medida que el terreno presionaba el revestimiento, el confinamiento del terreno aumentaba, y esta circunstancia ayudaba a inhibir o retrasar el proceso de físico-químico de hidratación de la anhidrita, lo que provocaba que la expansión se detuviese (lo que confirma la teoría de Wittke).

En las actuales líneas de alta velocidad españolas, dependientes de ADIF (Administración de Infraestructuras
Ferroviarias), pueden citarse diversos ejemplos de secciones tipo adecuadas para los fenómenos de hinchamiento que potencialmente puede presentar la anhidrita. El ejemplo más significativo es el de los Túneles de Montblanc, de la Línea de Alta Velocidad Madrid-Zaragoza-Barcelona-Frontera Francesa. En estos túneles, que incluye el mencionado de Lilla, además de los de Camp Magré y Puig Cabrer, se localizaban materiales del Terciario, constituidos por margas y argilitas yesíferas. En estos túneles las hipótesis sobre el desarrollo de fenómenos expansivos debidos a la presencia de anhidrita provocaron el desarrollo de fuertes presiones sobre la solera $(4,50 \mathrm{MPa})$ y sobre la bóveda $(2,5 \mathrm{MPa})$. Esta circunstancia condujo al diseño de una nueva sección tipo de geometría circular, con un revestimiento armado de hormigón HA-80 autocompactante, con una cuantía de acero de unos $10.000 \mathrm{~kg}$ por metro lineal de túnel, como se puede ver en la Figura 12. Se ejecutó una contrabóveda circular de canto variable desde $0,76 \mathrm{~m}$ en arranques de bóveda hasta 2,27 m en el punto inferior, y una bóveda circular de canto variable entre 0,46 y $0,76 \mathrm{~m}$, según el revestimiento existente. Tras diversos cálculos estructurales, se concluyó que la forma circular era la idónea a efectos de capacidad resistente, además de ser la más adecuada para casos en los que no es posible saber "a priori" qué zona se verá afectada por el hinchamiento.

En los túneles gemelos de Isuzkitza de la Autopista Vitoria-Éibar, tramo Arlabán-Eskoriatza, la sección de refuerzo en los tramos de anhidrita consiste en una contrabóveda circular armada de canto variable, desde $0,67 \mathrm{~m}$ en arranques de bóveda, hasta 2,27 m en el punto inferior, y una bóveda circular armada de 0,67 m de espesor, como se puede ver en la Figura 13. La cuantía de acero, compuesta de barras de diámetro 12, 16, 25 y $32 \mathrm{~mm}$ de

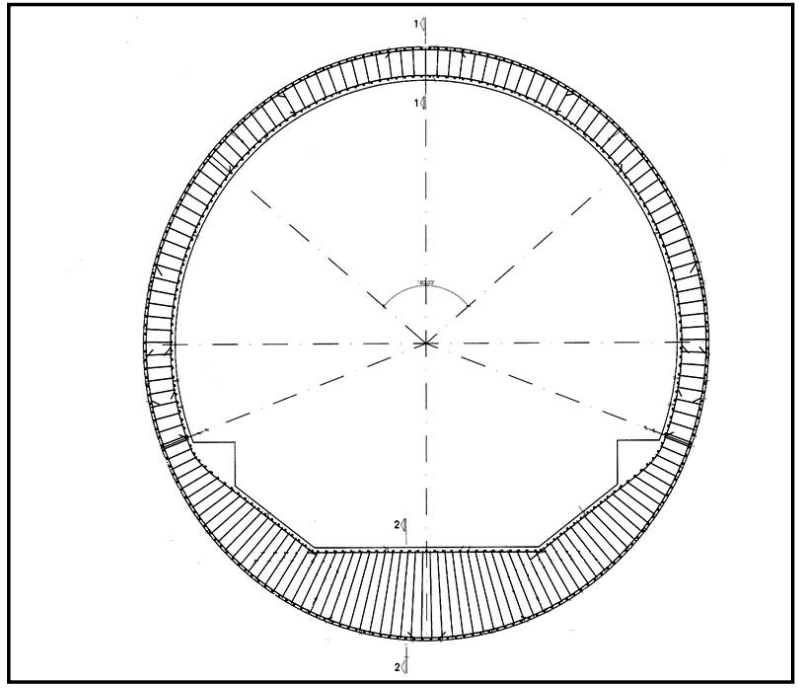

Figura 12. Sección tipo adoptada en el túnel de Lilla de la LAV Madrid-Zaragoza-Barcelona-frontera francesa. 


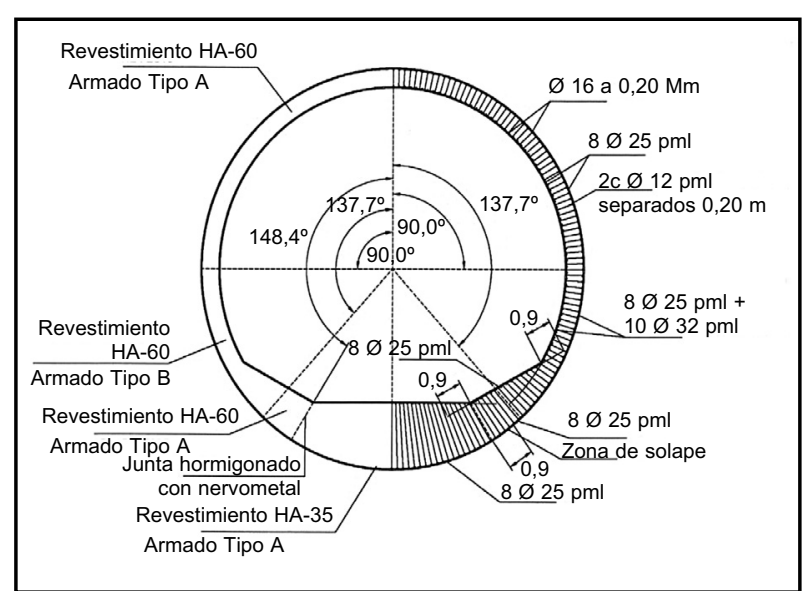

Figura 13. Sección tipo adoptada en los túneles de Isuzkitza de la Autopista Vitoria-Eibar.

acero B500S, es de $4.932 \mathrm{~kg} / \mathrm{m}$ túnel en contrabóveda y $2.952 \mathrm{~kg} / \mathrm{m}$ túnel en bóveda, con lo que la cantidad total de acero en túnel asciende a $7.884 \mathrm{~kg}$ de acero por metro lineal. El hormigón a emplear en la bóveda es de alta resistencia a compresión simple (de $60 \mathrm{MPa}$ ). En la contrabóveda se ha previsto un HA-35.

Otro ejemplo es el del Túnel de Fabares, en el tramo Lieres-Villaviciosa de la Autovía del Cantábrico. A este túnel se le dotó de una sección tipo similar, con una sección tipo dotada de una potente contrabóveda y un revestimiento armado, tal como se observa en la Figura 14.

Como puede comprobarse en los ejemplos anteriores, la tendencia actual es la de ejecutar revestimientos de hormigón armado muy pesados y rígidos, fuertemente armados.

\section{CONCLUSIONES}

El fenómeno de hinchamiento, muy extendido en los suelos arcillosos, también se observa en algunos tipos de rocas sulfatadas que presentan algunas características particulares en cuanto a su mineralogía, compuestas por arcillas expansivas y por sulfatos cálcicos anhidros. No obstante, la experiencia indica que no todos los túneles construidos en estos materiales anhidríticos $y / 0$ yesíferos sufren fenómenos expansivos.

El principal riesgo de la presencia de anhidrita en el túnel viene derivado del potencial hinchamiento de esta mineralogía, en presencia de agua, transformándose a yeso. Sin embargo, las razones por las cuales se desencadena la expansión, a nuestro juicio, no están claras.

Así, un macizo rocoso puede hinchar por dos motivos: o bien por contener materiales intrínsecamente expansivos,

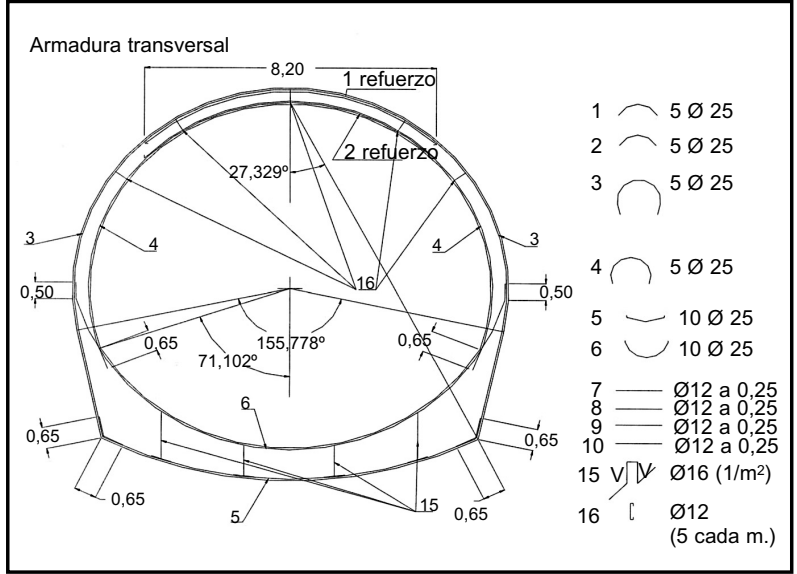

Figura 14. Sección tipo adoptada en los túneles de Fabares de la Autovía del Cantábrico.

o bien por contener sales disueltas en agua y precipitar el yeso como cristales en las fisuras. Además, no solo es necesario tener un material potencialmente expansivo para producir hinchamientos, sino también agua que acceda de forma continua para producir la hidratación necesaria.

Por otra parte, la distinción entre "rocas sulfatadas" y "suelos arcillosos" (arcillas y margas) pone de manifiesto que, bajo condiciones de construcción y servicio similares, el hinchamiento libre y las presiones de hinchamiento en rocas sulfatadas pueden ser un orden de magnitud mayores que en los materiales arcillosos. Además, parece ser que el hinchamiento en túneles excavados en rocas sulfatadas crece continuamente $y$, en algunos casos, no se puede definir el valor máximo de dichas presiones de hinchamiento.

El estudio comparativo realizado en este artículo, basado en una exhaustiva revisión de la bibliografía disponible, indica que el rango de valores de las presiones de hinchamiento medidas, realmente, en túneles construidos en estos materiales, han arrojado valores que oscilan entre mínimos prácticamente nulos a máximos de 5,5 Mpa (registrado en el Túnel de Wagenburg). Son las rocas sulfatadas de las facies Keuper y Muschelkalk del periodo Triásico, del sur de Alemania y norte de Suiza las que, hasta la fecha, han causado las principales patologías de daños y roturas en los túneles construidos. En España, el caso más importante se ha dado en el mencionado Túnel de Lilla, excavado en rocas blandas arcillosas sulfatadas de la cuenca terciaria del río Ebro, en el que la presión máxima de hinchamiento registrada hasta la fecha ha sido de $5 \mathrm{MPa}$.

En nuestra opinión, creemos que los dos aspectos más interesantes de estos problemas asociados al fenómeno de la expansividad de materiales en túneles construidos 
en anhidrita, en los que se deberían centrar y evolucionar futuros estudios, son: por una parte, la forma súbita como suelen manifestarse $y$, por otra, la manera incontrolada como suelen evolucionar en el tiempo.

Por otra parte, no cabe duda de que la forma geométrica de la sección del túnel y el método de excavación empleado juegan un papel importante en el fenómeno del hinchamiento.

Las deformaciones en un túnel por efecto del hinchamiento se producen frecuentemente con el levantamiento de la solera y las convergencias de los hastiales. Debido a la heterogeneidad intrínseca de las rocas sulfatadas, es frecuente que exista una gran anisotropía en el macizo y que, por tanto, los desplazamientos y las presiones tengan lugar de manera muy heterogénea. La rotura de los elementos estructurales aparece, especialmente, en la solera, aunque la casuística de los efectos del hinchamiento sobre el revestimiento del túnel es amplia y compleja.

En lo que concierne al revestimiento del túnel, a nuestro juicio, creemos que de las diversas soluciones de refuerzo empleadas, la que mejor resultado ha presentado es la colocación de revestimientos muy rígidos de hormigón, dotados de contrabóvedas muy robustas y fuertemente armadas, con elevadas cuantías de acero (de hasta incluso $10.000 \mathrm{~kg}$ por metro lineal de túnel, como en el caso del túnel de Lilla). Estos revestimientos adquieren unas cargas notables por lo que, lógicamente, deben estar diseñados para soportarlas. Además, se ha comprobado que a medida que el terreno presiona el revestimiento, aumenta el confinamiento del macizo, aspecto que ayuda a inhibir o retrasar el proceso de físico-químico de hidratación de la anhidrita, lo que provoca que la expansión se detenga, en algunos casos.

En nuestra opinión, también creemos que es de vital importancia el control de la circulación de agua en este tipo de materiales. El túnel se convierte en un canalizador de aguas que atraviesa el macizo y, por tanto, se cambia el régimen de circulación natural de las aguas subterráneas. La impermeabilización de la sección no siempre evita el desarrollo de fenómenos expansivos aunque, en otros casos, esa misma impermeabilización contribuye a evitar el hinchamiento del macizo. Así, pensamos que si no resulta posible deprimir el nivel freático e impermeabilizar la zona de manera que la presencia de agua no afecte a estos materiales, sería aconsejable estudiar otras posibles soluciones tendentes a minimizar el inicio y la posterior evolución del fenómeno expansivo (como, por ejemplo, la ejecución de un bulonado radial del túnel, en la zona potencialmente afectada).

Por último, hay que considerar la agresividad de estas aguas cargadas en sulfatos sobre los cementos y hormigones del túnel, por lo que el tipo de cemento a utilizar debe ser resistente a las aguas sulfatadas.

\section{BIBLIOGRAFÍA / BIBLIOGRAPHY}

(1) Amstad, C.; Kovari, K.: "Untertagbau in quellfähigem" Fels. Forschungsbericht 482 (2001) Vereinigung Schweizerisscher StraBenfachleute, 226 s., Zürich, German.

(2) Rauh, F.; Spaun, G.; Thuro, K.: "Assesment of the swelling potential of anhydrite in tunneling projects", The Geological Society of London. IAEG Engineering geology for tomorrow's cities. Proceedings of the 10th IAEG International Congress, Nottingham, United Kingdom, Paper number 473 (2006).

(3) Murray, R.: "Origin and diagénesis of gypsum and anhydrite". Journal of sedimentary geology, 34 (3) (1964) 512-523, Tulsa.

(4) Ortí Cabo, F.; Bayo, A.: "Características litoestratigráficas del Triásico Medio y Superior en el Baix Ebre (Tarragona-España)". Cuadernos de Geología Ibérica, vol. 4 (1977), pp. 223-238.

(5) Pina et al.: Epitaxial growth of gypsum on anhidrite: in situ AFM observations and computer calculations, Cambridge Publications (2000).

(6) Alonso, E. E.; Berdugo I. R.; Tarragó, D.; Ramón, A.: "Tunnelling in sulphate claystones", Keynote Paper. Proc. 14th European Conference on Soil Mechanics and Geotechnical Engineering, Madrid, Cuéllaret et al. (eds.), Millpress, Rottherdam, vol. 1 (2007), pp. 103-122.

(7) Tarragó Munté, D.: Degradación mecánica de argilitas sulfatadas y su efecto sobre la expansividad. Tesis de Licenciatura en Ingeniería Geológica. UPC, 120 (2006), p. 29.

(8) Huder, J.; Amberg, G.: "Quellung in Mergel, Opalinus-ton und Anhydrit". Schweizer. Bauzeit, 83. (1970), pp. 975-980.

(9) Steiner, W.: "Swelling rock in tunnels: rock characterization, effect of horizontal stresses and construction procedures". Int. J. Rock Mech. Min. Sci. \& Geoch. Abstr., vol. 30, no 4 (1993), pp. 361-380. http: //dx.doi.org/10.1016/0148-9062(93)91720-4

(10) Berdugo, I.; Alonso, E.; Gens, A.; Romero, E.: "Extreme expansive phenomena in a high-speed railway tunnel". Sesión 3. Avances Tecnológicos e Innovación. Congreso Internacional AETOS (2007), Madrid. 
(11) Henke K. F.; Kaiser W.; Nagel D.: "Geomechanische Unter- suchungen im Gipskeuper Forschungsberichte über Durchführung eines felsmechanischen Grossuersuches in der Nordróhre des Wagen-burgtunnels in Stuttgart". Strassenbau und Strassenverkehrstechnik, vol. 184, (1975), pp. 149-162.

(12) Serrano, A.; Oteo, C.; Dapena, E.; Martín Viñas, J.: "Análisis de los fenómenos de expansión de una formación de margas yesíferas". Bol. Inf. Lab. Carret. y Geot., 146 (1981), pp. 9-16.

(13) Serrano, A. A.; Dapena, J. E.; García de Paredes, I.: "Study on the swelling potencial of a gypsiferous marl mass in order to build the foundation of a power station", Proc. Rock mechanics and power plants, Romana (ed.), Balkema (1988), pp. 119-124.

(14) Lloret, A.; Alonso, E. E.; Gens, A.; Batlle, F.: "Analysis of a foundation on swelling rock. Rock Mechanics and Power Plants", ISRM Symposium. M. Romana, ed. Balkema, vol. 2 (1988).

(15) Alonso, E. E.: Alcoverro, J.: "Swelling and degradation of agillaceous rocks. Keynote lecture", Proc 3rd Int. Conf. Unsat. Soils, Recife, Balkema (2004), pp. 951-969.

(16) Alonso, E. E.; Berdugo, I.; Gens, A.; Romero, E.: "Expansive phenomena in an instrumented tunnel excavated in anhydritic-gypsiferous argillaceus rocks", Proceedings of the ISRM regional symposium EUROCK 2004 \& 53rd Geomechanics Colloquy. Edited by W. Schubert, VerlagGlückauf GmbH, Essen (2004), pp. 241-244.

(17) Alonso, E. E.; Berdugo, I.: "Expansive Behaviour of Sulphate-Bearing Clays. Keynote Lecture", Proc. Int. Conf. on Problematic Soils, Eastern Mediterranean University, Famagusta, N. Cyprus, vol. 2 (2005), pp. 477-498.

(18) Alonso, E. E.; Gens, A.; Berdugo, I.; Romero, E.: "Expansive behaviour of a sulphated clay in a railway tunnel", Proceedings of the 16 ${ }^{\text {th }}$ International Conference on Soils Mechanics and Geotechnical Engineering, Millpress, Rottherdam, vol. 3 (2005), pp. 1583-1586.

(19) Deu, A.; Tarragó, D.: Certamen Arquimedes 2004. Ministerio de Educación y Ciencia de España.

(20) Tarragó, D.; Deu, A.; Berdugo, I.; Melgarejo, J. C.; Tauler, E.: "Sobre la expansión en argilitas sulfatadas", en Cabalero, N., Cingolani, C. A., Linares, E., López de Luchi, M. G., Ostera, H. A. y Panarello, H. D. (eds.), Actas del XV Congreso Geológico Argentino no 786. (2005) p. 8.

(21) Alonso, E. E.; Berdugo, I.: "Degradation and swelling of sulphate-bearing claystones", Keynote Paper. Proc. VI CSAMR, Cartagena de Indias. Montero \& Colmenares (eds.) (2006), pp. 211-248.

(22) Deu Lozano, A.: "Estudio experimental del crecimiento de minerales sulfatados en argilitas anhidrítico-yesíferas". Tesina d'Especialitat de Enginyeria del Terreny, Cartogràfica i Geofísica. Tutores: Romero E. y Berdugo, I. Facultad de Geología. Universidad Politécnica de Cataluña (2008).

(23) Wittke, W.: "Stability Analysis for Tunnels". WBI Print 4. Chapter 3. Gliickauf (2000), p. 415.

(24) Wittke, W.: "Begrenzung der Quelldriicke durch Selbstabdichtung deim Tunnelbau im anhydrifiihrenden Gebirge". WBI Print 13.2. vols. (2003) Gluckauf, p. 112.

(25) Kovari, K.; Amstad, Ch.; Anagnostou, G.: "Design/construction methods - Tunneling in swelling rocks". Key questions in Rock Mechanics. Cundal et al. (eds.). (1988). Balkema, Rotterdam. 